Bull. Korean Math. Soc. 51 (2014), No. 3, pp. 823-829

http://dx.doi.org/10.4134/BKMS.2014.51.3.823

\title{
SOME CLASSIFICATIONS OF RULED SUBMANIFOLDS
}

\author{
Dong-Soo Kim, Young Ho Kim, And Sun Mi Jung
}

\begin{abstract}
Ruled submanifolds in Euclidean space satisfying some algebraic equations concerning the Laplace operator related to the isometric immersion and Gauss map are studied. Cylinders over a finite type curve or generalized helicoids are characterized with such algebraic equations.
\end{abstract}

\section{Introduction}

The theory of minimal submanifolds is still a very interesting subject in differential geometry from various points of view. As of minimal surfaces, it is well known that the only minimal ruled surfaces in Euclidean 3-space is part of the plane or the helicoid by the theorem of Catalan. This notion was generalized by the theory of ruled submanifolds in a Riemannian manifold in such a way that they are defined by a foliation of totally geodesic submanifolds of a given Riemannian manifold. In particular, if the ambient manifold is Euclidean, it is more interesting. The minimal ruled submanifold in Euclidean space was independently studied by Lumiste ([11]) and Barbosa et al. ([3]). They showed that a minimal ruled submanifold of Euclidean space is part of the plane or the generalized helicoid up to rigid motion parameterized by

$$
\begin{aligned}
x\left(s, t_{1}, \ldots, t_{n}\right)= & \left(t_{1} \cos \left(a_{1} s\right), t_{1} \sin \left(a_{1} s\right), \ldots,\right. \\
& \left.t_{k} \cos \left(a_{k} s\right), t_{k} \sin \left(a_{k} s\right), t_{k+1}, \ldots, t_{n}, b s\right),
\end{aligned}
$$

where $a_{1}, a_{2}, \ldots, a_{k}$ and $b$ are real numbers.

On the other hand, a minimal submanifold $M$ in Euclidean space $\mathbb{E}^{m}$ with the isometric immersion $x: M \rightarrow \mathbb{E}^{m}$ is characterized by their immersions and the Laplace operator $\Delta$ defined on them, namely, $\Delta x=0$. Generalizing this, Takahashi showed: Let $x: M \rightarrow \mathbb{E}^{m}$ be an isometric immersion of a

\footnotetext{
Received April 23, 2013.

2010 Mathematics Subject Classification. 53A04, 53A05, 53A07.

Key words and phrases. Gauss map, ruled submanifold, Grassmannian manifold.

The first author was supported by Basic Science Research Program through the National Research Foundation of Korea (NRF) funded by the Ministry of Education, Science and Technology (2010-0022926).

The second author was supported by Basic Science Research Program through the National Research Foundation of Korea (NRF) funded by the Ministry of Education, Science and Technology (2012R1A1A2042298).
} 
Riemannian manifold $M$ into the Euclidean space $\mathbb{E}^{m}$. If $\Delta x=\lambda x(\lambda \neq 0)$ holds, then $M$ is a minimal submanifold in a hypersphere of Euclidean space $([12])$.

Extending this point of view, in the late 1970's by using the spectral decomposition Chen introduced the notion of finite type smooth map on Riemannian manifolds in Euclidean space $([4,5])$. A smooth map $\Phi$ on a Riemannian manifold $M$ into an $m$-dimensional Euclidean space $\mathbb{E}^{m}$ is said to be of finite type if $\Phi$ can be expressed as a finite sum of eigenvectors of the Laplacian $\Delta$ of $M$, that is, $\Phi=\Phi_{0}+\sum_{i=1}^{k} \Phi_{i}$, where $\Phi_{0}$ is a constant map, $\Phi_{1}, \ldots, \Phi_{k}$ non-constant maps such that $\Delta \Phi_{i}=\lambda_{i} \Phi_{i}, \lambda_{i} \in \mathbb{R}, i=1,2, \ldots, k([4,5])$. Furthermore, $M$ is said to be of $k$-type if all eigenvalues $\lambda_{1}, \lambda_{2}, \ldots, \lambda_{k}$ are mutually different. In particular, null 1-type submanifolds in Euclidean space are minimal and null 1-type smooth vector fields are also said to be harmonic.

In this regards, Dillen ([7]) showed that an $n$-dimensional ruled submanifold in $\mathbb{E}^{m}$ of finite type immersion is part of either a cylinder on a curve of finite type or the generalized helicoid.

On the other hand, in [1] Baikoussis proved that an $n$-dimensional ruled submanifold $M$ in $\mathbb{E}^{m}$ with finite type Gauss map is part of an $n$-plane. In the case, the Gauss map $G$ is in fact harmonic, that is, $\Delta G=0$.

However, if the Laplacian of each component $X_{A}(A=1,2, \ldots, m)$ of a certain vector field $X$ in $\mathbb{E}^{m}$ is a linear function in $X_{1}, X_{2}, \ldots, X_{m}$, it is not of finite type in general. In other words, it has the form

$$
\Delta X=A X+\mathbf{b}
$$

for some $m \times m$-matrix $A$ and a constant vector $\mathbf{b}$ (cf. $[2,8,9]$ ).

In this article, we study the ruled submanifold $M$ in the Euclidean space $\mathbb{E}^{m}$ satisfying the equation

$$
\Delta x=A x+\mathbf{b} \quad \text { and } \quad \Delta G=A G+\mathbf{b}
$$

for some $m \times m$-matrix $A$ and a constant vector $\mathbf{b}$, where $x$ is the isometric immersion of $M$ into $\mathbb{E}^{m}$ and $G$ is the Gauss map defined on $M$.

All of geometric objects under consideration are smooth and submanifolds are assumed to be connected unless otherwise stated.

\section{Preliminaries}

Let $M$ be an $n$-dimensional Riemannian manifold isometrically immersed into an $m$-dimensional Euclidean space $\mathbb{E}^{m}$ via the immersion $x$.

Let $\left(x_{1}, x_{2}, \ldots, x_{n}\right)$ be a local coordinate system of $M$ in $\mathbb{E}^{m}$. For the components $g_{i j}$ of the metric $\langle\cdot, \cdot\rangle$ on $M$ induced from that of $\mathbb{E}^{m}$, we denote by $\left(g^{i j}\right)$ (respectively, $\mathcal{G}$ ) the inverse matrix (respectively, the determinant) of the matrix $\left(g_{i j}\right)$. Then, the Laplacian $\Delta$ on $M$ is given by

$$
\Delta=-\frac{1}{\sqrt{|\mathcal{G}|}} \sum_{i, j} \frac{\partial}{\partial x_{i}}\left(\sqrt{|\mathcal{G}|} g^{i j} \frac{\partial}{\partial x_{j}}\right) .
$$


We now choose an adapted local orthonormal frame $\left\{e_{1}, e_{2}, \ldots, e_{m}\right\}$ in $\mathbb{E}^{m}$ such that $e_{1}, e_{2}, \ldots, e_{n}$ are tangent to $M$ and $e_{n+1}, e_{n+2}, \ldots, e_{m}$ normal to $M$. The Gauss map $G: M \rightarrow G(n, m) \subset \mathbb{E}^{N}\left(N={ }_{m} C_{n}\right), G(p)=\left(e_{1} \wedge e_{2} \wedge\right.$ $\left.\cdots \wedge e_{n}\right)(p)$, of $x$ is a smooth map which carries a point $p$ in $M$ to an oriented $n$-plane in $\mathbb{E}^{m}$ which is obtained from the parallel translation of the tangent space of $M$ at $p$ to an $n$-plane passing through the origin in $\mathbb{E}^{m}$, where $G(n, m)$ is the Grassmannian manifold consisting of all oriented $n$-planes through the origin of $\mathbb{E}^{m}$.

An inner product $\ll \cdot, \cdot \gg$ on $G(n, m) \subset \mathbb{E}^{N}$ is defined by

$$
\ll e_{i_{1}} \wedge \ldots \wedge e_{i_{n}}, e_{j_{1}} \wedge \cdots \wedge e_{j_{n}} \gg=\operatorname{det}\left(\left\langle e_{i_{l}}, e_{j_{k}}\right\rangle\right) .
$$

Then, $\left\{e_{i_{1}} \wedge e_{i_{2}} \wedge \cdots \wedge e_{i_{n}} \mid 1 \leq i_{1}<\cdots<i_{n} \leq m\right\}$ is an orthonormal basis of $\mathbb{E}^{N}$. by

We now consider a ruled submanifold $M$ over the base curve $\alpha$ parameterized

$$
x=x\left(s, t_{1}, t_{2}, \ldots, t_{r}\right)=\alpha(s)+\sum_{i=1}^{r} t_{i} e_{i}(s), \quad s \in I, t_{i} \in I_{i},
$$

where $I_{i}$ 's are some open intervals for $i=1,2, \ldots, r$.

Here, we may assume that the base curve $\alpha$ is of unit speed and

$$
\left\langle\alpha^{\prime}(s), e_{i}(s)\right\rangle=0, \quad\left\langle e_{i}(s), e_{j}(s)\right\rangle=\delta_{i j} \quad\left\langle e_{i}^{\prime}(s), e_{j}(s)\right\rangle=0
$$

for $i, j=1,2, \ldots, r$.

For each $s$, let $E(s, r)$ be an open subset of $\operatorname{Span}\left\{e_{1}, e_{2}, \ldots, e_{r}\right\}$, the linear span of $e_{1}, e_{2}, \ldots, e_{r}$. We call $E(s, r)$ the rulings of $M$. In particular, the ruled submanifold $M$ is said to be cylindrical if $E(s, r)$ is parallel along $\alpha$, or non-cylindrical otherwise.

\section{Ruled submanifolds satisfying $\Delta x=A x+b$}

Let $M$ be an $(r+1)$-dimensional ruled submanifold in $\mathbb{E}^{m}$ with the base curve $\alpha$. Without loss of generality, we may assume that $\alpha$ is a unit speed curve, that is, $\left\langle\alpha^{\prime}(s), \alpha^{\prime}(s)\right\rangle=1$. From now on, the prime' denotes $d / d s$ unless otherwise stated. We may also choose orthonormal vector fields $e_{1}(s), \ldots, e_{r}(s)$ generating the rulings along $\alpha$ with

$$
\left\langle\alpha^{\prime}(s), e_{i}(s)\right\rangle=0,\left\langle e_{i}^{\prime}(s), e_{j}(s)\right\rangle=0, \quad i, j=1,2, \ldots, r .
$$

A parametrization of $M$ is given by

$$
x=x\left(s, t_{1}, t_{2}, \ldots, t_{r}\right)=\alpha(s)+\sum_{i=1}^{r} t_{i} e_{i}(s) .
$$

We now consider the case that the ruled submanifold $M$ is cylindrical. Then, we may take the generators $e_{1}, e_{2}, \ldots, e_{r}$ of the rulings $E(s, r)$ as constant vector fields. 
Suppose $M$ satisfies the equation

$$
\Delta x=A x+\mathbf{b}
$$

for some $m \times m$-matrix $A$ and a constant vector $\mathbf{b}$. Then, by Cayley-Hamilton's Theorem, there exist some constants $c_{0}, c_{1}, \ldots, c_{m}$ such that

$$
A^{m}+c_{o} A^{m-1}+\cdots+c_{m-1} A+c_{m} I=0,
$$

where $I$ denotes the identity matrix of degree $m$. Using (3.3), we have

$$
\Delta^{m+1} x+c_{0} \Delta^{m} x+\cdots+c_{m} \Delta x=0 .
$$

According to Proposition 4.1 in [6], the base curve $\alpha$ is of finite type. Therefore, we have:

Theorem 3.1. Let $M$ be a cylindrical ruled submanifold of $\mathbb{E}^{m}$ satisfying $\Delta x=$ $A x+\boldsymbol{b}$ for some $m \times m$-matrix $A$ and a constant vector $\boldsymbol{b} \in \mathbb{R}^{m}$. Then, $M$ is a cylinder over the finite type base curve.

Next, consider the case that $M$ is non-cylindrical.

If we define a function $q$ on $M$ by

$$
q=\left\|x_{s}\right\|^{2}=1+2 \sum_{i=1}^{r} t_{i}\left\langle\alpha^{\prime}, e_{i}^{\prime}\right\rangle+\sum_{i, j=1}^{r} t_{i} t_{j}\left\langle e_{i}^{\prime}, e_{j}^{\prime}\right\rangle,
$$

then the Laplacian $\Delta$ of $M$ is given by

$$
\Delta=\frac{1}{2 q^{2}} \frac{\partial q}{\partial s} \frac{\partial}{\partial s}-\frac{1}{q} \frac{\partial^{2}}{\partial s^{2}}-\frac{1}{2 q} \sum_{i=1}^{r} \frac{\partial q}{\partial t_{i}} \frac{\partial}{\partial t_{i}}-\sum_{i=1}^{r} \frac{\partial^{2}}{\partial t_{i}^{2}} .
$$

The function $q$ is a polynomial in $t=\left(t_{1}, t_{2}, \ldots, t_{r}\right)$ with functions of $s$ as coefficients. Since $M$ is non-cylindrical, we may assume that $q$ has degree 2 in $t$ without loss of generality. Then, we can easily have:

Lemma $3.1([7])$. Let $M$ be a non-cylindrical ruled submanifold in $\mathbb{E}^{m}$ parametrized by (3.2). If $P$ is a polynomial with functions in $s$ as coefficients and $\operatorname{deg}(P)=d$, then

$$
\Delta\left(\frac{P(t)}{q^{m}}\right)=\frac{\tilde{P}(t)}{q^{m+3}},
$$

where $\tilde{P}$ is a polynomial in $t$ with functions in $s$ as coefficients and $\operatorname{deg}(\tilde{P}) \leq$ $d+4$.

We now suppose that $M$ satisfies (3.3) for a non-trivial matrix $A$. Then, for some constant $c_{0}, c_{1}, \ldots, c_{m}$, we have

$$
\Delta^{m+1} x+c_{0} \Delta^{m} x+\cdots+c_{m} \Delta x=0 .
$$

Let $x_{A}$ be the $A$-th component of $x$ in $\mathbb{E}^{m}$, where $A=1,2, \ldots, m$. Then,

$$
\Delta x_{A}=\frac{Q_{A}(t)}{q^{2}}
$$


for some polynomial $Q_{A}(t)$ in $t=\left(t_{1}, t_{2}, \ldots, t_{r}\right)$ with $\operatorname{deg} Q_{A}(t) \leq 5$. By applying Lemma 3.2, we have

$$
\Delta^{j} x_{A}=\frac{Q_{A j}(t)}{q^{3 j-1}}
$$

with $\operatorname{deg} Q_{A j} \leq 1+4 j, j=1,2, \ldots$ If $j$ goes up by one, the degree of numerator of $\Delta^{j} x_{A}$ goes up by at most 3 while that of the denominator goes up by 4 . Thus, for some positive integer $i, \Delta^{i+1} x+\lambda_{1} \Delta^{i} x+\cdots+\lambda_{i} \Delta x=0$ never occurs unless $\Delta x=0$, that is, $M$ is minimal. Therefore, the matrix $A$ must be zero. Hence, we have:

Theorem 3.2. Let $M$ be a non-cylindrical ruled submanifold of $\mathbb{E}^{m}$ satisfying $\Delta x=A x+\boldsymbol{b}$ for some $m \times m$-matrix $A$ and a constant vector $\boldsymbol{b} \in \mathbb{R}^{m}$. Then, $M$ is minimal, i.e., $M$ is part of a plane or a generalized helicoid.

If we consider the result of [7], we have the following characterization of the ruled submanifold of finite type immersion.

Theorem 3.3. Let $x: M \rightarrow \mathbb{E}^{m}$ be an isometric immersion of ruled submanifold in $\mathbb{E}^{m}$. Then, $x$ is of finite type if and only if $M$ is part of a cylinder over a finite type curve or $x$ satisfies $\Delta x=A x+b$ for some $m \times m$-matrix $A$ and a constant vector $\boldsymbol{b} \in \mathbb{R}^{m}$.

\section{Ruled submanifolds satisfying $\Delta G=A G+\mathrm{b}$}

In this section, we always assume that the parametrization (3.2) satisfies the condition (3.1). Then, $M$ has the Gauss map

or, equivalently

$$
G=\frac{1}{\left\|x_{s}\right\|} x_{s} \wedge x_{t_{1}} \wedge \cdots \wedge x_{t_{r}},
$$

$$
G=\frac{1}{q^{1 / 2}}\left(\Phi+\sum_{i=1}^{r} t_{i} \Psi_{i}\right),
$$

where the vectors $\Phi$ and $\Psi_{i}$ are defined by

$$
\Phi=\alpha^{\prime} \wedge e_{1} \wedge \cdots \wedge e_{r} \text { and } \Psi_{i}=e_{i}^{\prime} \wedge e_{1} \wedge \cdots \wedge e_{r}
$$

for $i=1,2, \ldots, r$.

Now, we prove:

Theorem 4.1. The only ruled submanifolds of $\mathbb{E}^{m}$ with $\Delta G=A G+\boldsymbol{b}$ for some matrix $A$ and a vector $\boldsymbol{b}$ are parts of planes or cylinders over a curve of finite type.

Proof. Let $M$ be a cylindrical $(r+1)$-dimensional ruled submanifold parameterized by $(3.2)$ in $\mathbb{E}^{m}$ satisfying

$$
\Delta G=A G+\mathbf{b}
$$


for some $m \times m$-matrix $A$ and a constant vector $\mathbf{b}$. We may assume that $e_{1}, e_{2}, \ldots, e_{r}$ generating the rulings are constant vectors.

The Laplacian $\Delta$ of $M$ is then naturally expressed by

$$
\Delta=-\frac{\partial^{2}}{\partial s^{2}}-\sum_{i=1}^{r} \frac{\partial^{2}}{\partial t_{i}^{2}}
$$

and the Gauss map $G$ of $M$ is given by

$$
G=\alpha^{\prime} \wedge e_{1} \wedge \cdots \wedge e_{r} .
$$

If we denote by $\Delta^{\prime}$ the Laplacian of $\alpha$, that is $\Delta^{\prime}=-\frac{\partial^{2}}{\partial s^{2}}$, we have the Laplacian $\Delta G$ of the Gauss map

$$
\Delta G=\Delta^{\prime} \alpha^{\prime} \wedge e_{1} \wedge \cdots \wedge e_{r}
$$

Since (4.2) holds, we have (3.4) and thus we obtain

$$
\Delta^{m+1} G+c_{0} \Delta^{m} G+\cdots+c_{m} \Delta G=0,
$$

or, equivalently,

$$
\Delta^{\prime m+1} \alpha^{\prime}+c_{0} \Delta^{\prime k} \alpha^{\prime}+\cdots+c_{m} \Delta^{\prime} \alpha^{\prime}=0
$$

which implies that $\alpha^{\prime}(s)=a_{1}+a_{2} s+\sum_{i=1}^{k}\left\{\tilde{b}_{i} \cos \left(l_{i} s\right)+\tilde{c}_{i} \sin \left(l_{i} s\right)\right\}$ for some positive integer $k$, where $l_{1}<l_{2}<\cdots<l_{k}$ are positive real numbers and $a_{1}, a_{2}, \tilde{b}_{i}, \tilde{c}_{i}$ are vectors in $\mathbb{E}^{m}$ such that $b_{i}$ and $c_{i}$ are not simultaneously zero for each $i=1,2, \ldots, k$ (cf. [10]). Since we assume that the base curve $\alpha$ is of unit speed, the coefficient $a_{2}$ vanishes. Thus, we have $\alpha(s)=\tilde{a}_{0}+\tilde{a}_{1} s+$ $+\sum_{j=1}^{k}\left\{b_{i} \cos \left(l_{i} s\right)+c_{i} \sin \left(l_{i} s\right)\right\}$ where $\tilde{a}_{0}, \tilde{a}_{1}, \tilde{a}_{2}, b_{i}, c_{i}$ are vectors in $\mathbb{E}^{m}$ such that $b_{i}$ and $c_{i}$ are not simultaneously zero for each $i=1,2, \ldots, k$. This implies that the curve $\alpha$ is of finite type.

We now suppose that a non-cylindrical ruled submanifold $M$ satisfies $\Delta G=$ $A G+\mathbf{b}$ for some $m \times m$-matrix $A$ and a constant vector $\mathbf{b}$. Then we have the Laplacian

$$
\Delta=\frac{1}{2 q^{2}} \frac{\partial q}{\partial s} \frac{\partial}{\partial s}-\frac{1}{q} \frac{\partial^{2}}{\partial s^{2}}-\frac{1}{2 q} \sum_{i=1}^{r} \frac{\partial q}{\partial t_{i}} \frac{\partial}{\partial t_{i}}-\sum_{i=1}^{r} \frac{\partial^{2}}{\partial t_{i}^{2}}
$$

Similarly to obtain (3.4), we have

$$
\Delta^{m+1} G+c_{0} \Delta^{m} G+\cdots+c_{m} \Delta G=0
$$

for some constants $c_{0}, c_{1}, \ldots, c_{m}$.

Quite similarly as in [1], we get

$$
G=\frac{G_{0}(t)}{q^{1 / 2}}, \Delta G=\frac{G_{1}(t)}{q^{(1 / 2)+3}}, \ldots, \Delta^{j} G=\frac{G_{j}(t)}{q^{(1 / 2)+3 j}}, j=0,1,2, \ldots,
$$

where $G_{j}(t)$ is a polynomial in $t=\left(t_{1}, t_{2}, \ldots, t_{r}\right)$ with functions in $s$ as coefficients and $\operatorname{deg} G_{j}(t) \leq 1+4 j$. As before, if (4.5) holds, there exist no other cases but

$$
\Delta G=0
$$


If we follow along the argument in [1] with harmonic Gauss map, we obtain $M$ is part of an $(r+1)$-plane.

\section{References}

[1] C. Baikoussis, Ruled submanifolds with finite type Gauss map, J. Geom. 49 (1994), no. $1-2,42-45$.

[2] C. Baikoussis and D. Blair, On the Gauss map of ruled surfaces, Glasgow Math. J. 34 (1992), no. 3, 355-359

[3] J. M. Barbosa, M. Dajczer, and I. P. Jorge, Minimal ruled submanifolds in spaces of constant curvature, Indiana Univ. Math. J. 33 (1984), no. 4, 531-547.

[4] B.-Y. Chen, Total Mean Curvature and Submanifolds of Finite Type, World Scientific, Singapore, 1984.

[5] _ A report on submanifolds of finite type, Soochow J. Math. 22 (1996), 117-337.

[6] B.-Y. Chen and M. Petrovic, On spectral decomposition of immersion of finite type, Bull. Austral. Math. Soc. 44 (1991), no. 2, 117-129.

[7] F. Dillen, Ruled submanifolds of finite type, Proc. Amer. Math. Soc. 114 (1992), no. 3, 795-798.

[8] F. Dillen, J. Pas, and L. Verstraelen, On the Gauss map of surfaces of revolution, Bull. Inst. Math. Acad. Sinica 18 (1990), no. 3, 239-246.

[9] D.-S. Kim, On the Gauss map of quadric hypersurfaces, J. Korean Math. Soc. 31 (1994), no. $3,429-437$.

[10] D.-S. Kim and H.-S. Chung, Space curves satisfying $\Delta H=A H$, Bull. Korean Math. Soc. 31 (1994), no. 2, 193-200.

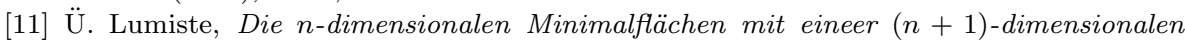
asymtotischen Richitung im jeden Punkute, Tarrtu Riikl. Ül. Toimetised 62 (1958), $117-141$.

[12] T. Takahashi, Minimal immersions of Riemannian manifolds, J. Math. Soc. Japan 18 (1966), 380-385.

DonG-SOO KIM

Department of Mathematics

Chonnam National University

KWANGJU 500-757, KorEA

E-mail address: dosokim@chonnam.ac.kr

Young Ho KIM

Department of Mathematics

KyungPoOK National University

TAEgu 702-701, Korea

E-mail address: yhkim@knu.ac.kr

Sun Mi Jung

Department of Mathematics

KyungPoOK National University

TAegu 702-701, Korea

E-mail address: jung3756@hanmail.net 